\title{
Polymorphic+Typeclass Superposition
}

\author{
Daniel Wand \\ Max-Planck-Institut für Informatik, Saarbruecken, DE. \\ dwand@mpi-inf .mpg. de \\ http://www.mpi-inf.mpg.de/ dwand/paar14/
}

\begin{abstract}
We present an extension of superposition that natively handles a polymorphic type system extended with type classes, thus eliminating the need for type encodings when used by an interactive theorem prover like Isabelle/HOL. We describe syntax, typing rules, semantics, the polymorphic superposition calculus and an evaluation on a problem set that is generated from Isabelle/HOL theories. Our evaluation shows that native polymorphic+typeclass performance compares favorably to monomorphisation, a highly efficient but incomplete way of dealing with polymorphism.
\end{abstract}

\section{Introduction}

Polymorphism is a prevalent feature in interactive theorem proving and functional programming. There is extensive research in encoding polymorphism into untyped or monomorphic form (e.g. [5, 9, 10]), in an effort to be able to use ATPs on polymorphic problems. Despite of this there are few automated theorem provers (ATPs) capable of natively supporting polymorphism. We are only aware of the SMT solver Alt-Ergo 8 .

Superposition and SMT based provers, even without supporting polymorphism, are successful in discharging goals arising in interactive theorem proving; [4 reports combined success rates of $60 \%$ on a set of Isabelle/HOL theories. In this abstract we report on work to bring rank-1 polymorphism extended with type classes [15] to superposition. We used TFF1 $[$ ] 's polymorphic semantic as a starting point for ours, and extended it with type classes. Our prototype implementation uses an extended version of SPASS's input syntax, which allows utilizing additional annotations described in [7].

\section{Declarations}

Notation We use $f$ for function symbols, $p$ for predicate symbols, $t, s$ for terms, $\phi$ for formulas, $u$ for term variables, $\sigma$ for substitutions, $\tau$ for types, $\kappa$ for type constructors, $\alpha$ for type variables and $c$ for type classes, $C$ for sets of type classes, $n$ for type arity and $m$ for term arity. We write $t[s]_{p}$ to denote a term, which is $t$ with the subterm at position $p$ of $t$ replaced by $s$.

Signature A polymorphic+typeclass first-order language signature is a tuple $\Sigma=(\mathcal{F}, \mathcal{P}, \mathcal{T}, \mathcal{T C})$ There must be exactly one declaration per function and predicate symbol.

$\mathcal{F}$ The elements f : $\forall \alpha_{1}: C_{1} \ldots \alpha_{n}: C_{n} \cdot \tau_{1} \ldots \tau_{m} \rightarrow \tau \in \mathcal{F}$ are function declarations.

We use the syntax $f<\tau_{f_{1}}, \ldots, \tau_{f_{n}}>\left(t_{1}, \ldots, t_{m}\right)$, to write a function term, denoting the appropriate instantiations of the $\alpha_{i}$ by the corresponding type argument $\tau_{f_{i}}$ and that of $\tau_{i}$ by the term argument $t_{i}$. With the declaration, the return type $\tau$ is derivable from this representation.

$\mathcal{P}$ The elements p : $\forall \alpha_{1}: C_{1} \ldots \alpha_{n}: C_{n} . \tau_{1} \ldots \tau_{m} \rightarrow o_{\mathcal{B}} \in \mathcal{P}$ are predicate declarations. Similar to functions we write $p<\tau_{p_{1}}, \ldots, \tau_{p_{n}}>\left(t_{1}, \ldots, t_{m}\right)$. 
$\mathcal{T}$ The elements $\forall \alpha_{1}: C_{1} \ldots \alpha_{n}: C_{n} . \kappa\left(\alpha_{i}, \ldots\right): c \in \mathcal{T}$ are type declarations.

There must be at most one declaration per type constructor $\kappa$ and $c$ and at least one per $\kappa$. $\kappa$ must always have the same arity. In order to allow $\kappa \mathrm{s}$, that are not part of any (custom) type class, there is the special type class $\emptyset$ that can be used in the required declaration and represents no type class. All $\kappa$ are part of this type class.

$\mathcal{T C}$ The elements $c_{1} \subseteq c_{2} \in \mathcal{T C}$ are subclass declarations.

Type terms Let $\mathcal{X}_{\tau}$ be a given countably infinite set of type variables. All type terms are recursively defined as:

- Every type variable in $\mathcal{X}_{\tau}$ is a type term

- For all $\forall \alpha_{1}: C_{1} \ldots \alpha_{n}: C_{n} . \kappa\left(\alpha_{i}, \ldots\right): c \in \mathcal{T}$ let $\tau_{1}, \ldots, \tau_{n}$ be type terms then $\kappa\left(\tau_{1}, \ldots, \tau_{n}\right)$ is also a type term

Terms Let $\mathcal{X}_{t}$ be a given countably infinite set of term variables. All, possibly ill-typed, terms are recursively defined as:

- Every term variable in $\mathcal{X}_{t}$ is a term

- For all $\mathrm{f}: \forall \alpha_{1}: C_{1} \ldots \alpha_{n}: C_{n} \cdot \tau_{1} \ldots \tau_{m} \rightarrow \tau \in \mathcal{F}$ let $t_{1}, \ldots, t_{m}$ be terms and $\tau_{1}, \ldots, \tau_{n}$ be type terms then $\mathrm{f}<\tau_{1}, \ldots, \tau_{n}>\left(t_{1}, \ldots, t_{m}\right)$ is also a term.

Formulas All, possibly ill-typed, formulas are recursively defined as:

1. For all $\mathrm{p}: \forall \alpha_{1}: C_{1} \ldots \alpha_{n}: C_{n} \cdot \tau_{1} \ldots \tau_{m} \rightarrow o_{\mathcal{B}} \in \mathcal{P}$ let $t_{1}, \ldots, t_{m}$ be terms and $\tau_{1}, \ldots, \tau_{n}$ be type terms, then $\mathrm{p}<\tau_{1}, \ldots, \tau_{n}>\left(t_{1}, \ldots, t_{m}\right)$ is a (atomic) formula.

2. Let $t_{1}, t_{2}$ be terms, then $t_{1} \approx t_{2}$ is a (atomic) formula.

3. Let $\phi_{1}, \phi_{2}$ be formulas, then $\phi_{1} \wedge \phi_{2}, \phi_{1} \vee \phi_{2}, \phi_{1} \Rightarrow \phi_{2}, \phi_{1} \Leftrightarrow \phi_{2}$ and $\neg \phi_{1}$ are also formulas.

4. Let $\phi$ be a formula, $u$ a fresh term variable and $\tau$ a type term, then $\forall u: \tau . \phi$ and $\exists u: \tau . \phi$ are also formulas.

5. Let $\phi$ be a formula, $\alpha$ a fresh type variable and $C$ an optional type class constraint (a set of type classes written as $c_{1} \& \ldots \& c_{n}$ ), then $\forall_{\text {types }} \alpha: C$. $\phi$ is also a formula.

Note that both quantifiers in 4 , range over term variables. The quantifier in 5 , ranges over type variables and its existential equivalent is not well typed, since existential types are not supported by this formalization.

\section{Syntax}

We extended the monomorphic SPASS syntax to support our polymorphic+typeclass calculus. We show here one simple example file and make the complete specification available on the abstracts webpage. Every problem file must begin with, where example can be replaced by an other string:

begin_problem (example).

Continuing with the preamble where additional information may be entered inbetween $*$ and $*$.

list_of_descriptions.

name $(\{* *\})$.

author $(\{* *\})$. 
status (unknown).

description $(\{* *\})$.

end_of_list.

Next the used symbols must be defined with their arity. Where a single number describes the term arity and where number1+number 2 describes the type arity to be number 1 and the term arity to be number2.

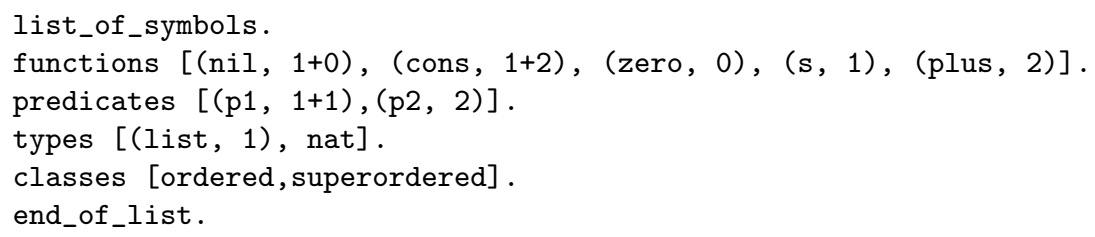

Now the declarations described in the previous section follow:

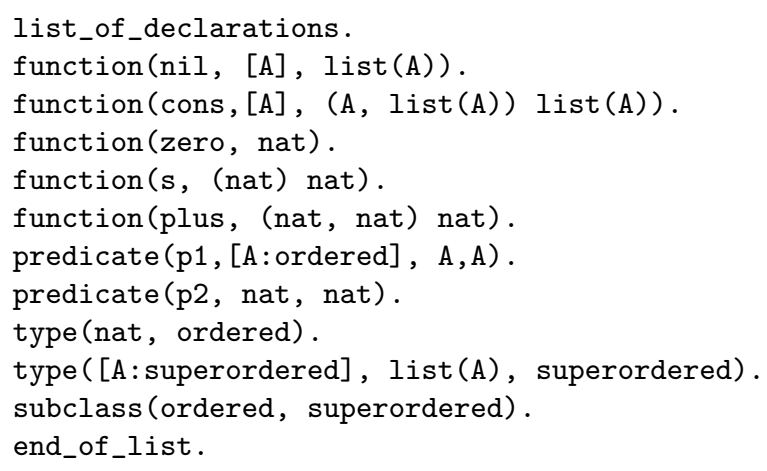

The set of axioms:

list_of_formulae(axioms).

formula (forall ( $[X:$ nat $]$, equal (plus (zero,X),X)), plus_zero).

formula (forall ( $[X$ :nat, Y:nat $]$, equal: $\operatorname{lr}(p \operatorname{lus}(s(X), Y), s(p l u s(X, Y)))$ ), plus_s).

formula (forall_types ( $[A]$, forall $([X: A, Y: A], p 1<A>(X, Y))), p 1)$.

end_of_list.

And the conjecture:

list_of_formulae(conjectures).

formula (forall ( $[X:$ nat $]$, equal (plus (zero,X),X)), conjecture).

end_of_list.

\section{Typing Rules}

Let $\gamma_{\mathcal{C}}^{\mathcal{T}}$ represent a typing context, with $\mathcal{T}$ as mapping from term variables to types or to a special value denoting "undefined" and $\mathcal{C}$ as mapping from type variables to a sets of type classes or to "undefined". All well-typedness assertions are implicitly relative to a given signature. 
Type Classes Type classes represent sets of types. A set of type classes corresponds to the intersection of the sets the individual type classes represent.

$$
\begin{array}{cc}
\overline{\mathcal{C} \vdash \tau: \emptyset} \text { (empty) } & \overline{\mathcal{C} \vdash \alpha: \mathcal{C}(\alpha)} \text { (type var) } \\
\frac{\forall \alpha_{1}: \mathrm{C}_{1} \ldots \alpha_{\mathrm{n}}: \mathrm{C}_{\mathrm{n}} . \kappa\left(\alpha_{\mathrm{i}}, \ldots\right): \mathrm{c} \in \mathcal{T} \quad \mathcal{C} \vdash \kappa\left(\alpha_{i}, \ldots\right) \sigma: C}{\mathcal{C} \vdash \kappa\left(\alpha_{i}, \ldots\right) \sigma: C \uplus\{c\}} & \mathcal{C} \vdash \alpha_{1} \sigma: C_{1} \quad \ldots \quad \mathcal{C} \vdash \alpha_{m} \sigma: C_{m} \\
\frac{\mathrm{c}_{1} \subseteq \mathrm{c}_{2} \in \mathcal{T C} \quad \mathcal{C} \vdash \tau: C \uplus\left\{c_{1}\right\}}{\mathcal{C} \vdash \tau \sigma: C \uplus\left\{c_{2}\right\}} \text { (subclass) }
\end{array}
$$

Terms A term is well-typed, for a given $\gamma_{\mathcal{C}}^{\mathcal{T}}$, if and only if a type can be derived by the following rules:

$$
\begin{gathered}
\frac{\overline{\gamma_{\mathcal{C}}^{\mathcal{T}} \vdash u: \mathcal{T}(u)} \text { (term var) }}{\mathrm{f}: \forall \alpha_{1}: \mathrm{C}_{1} \ldots \alpha_{\mathrm{n}}: \mathrm{C}_{\mathrm{n}} \cdot \tau_{1} \ldots \tau_{\mathrm{m}} \rightarrow \tau \in \mathcal{F} \quad \gamma_{\mathcal{C}}^{\mathcal{T}} \vdash t_{1}: \tau_{1} \sigma \quad \ldots \quad \gamma_{\mathcal{C}}^{\mathcal{T}} \vdash t_{n}: \tau_{n} \sigma} \\
\gamma_{\mathcal{C}}^{\mathcal{T}} \vdash f<\alpha_{1} \sigma, \ldots, \alpha_{m} \sigma>\left(t_{1}, \ldots, t_{n}\right): \tau \sigma
\end{gathered}
$$

and for all $i: 1 . . n . \mathcal{C} \vdash \alpha_{i} \sigma: C_{i}$

Formulas Without loss of generality this section assumes that all variables are named uniquely. It further assumes that $o_{\mathcal{B}}, o$ are types which do not match any type in the signature. We use two different boolean type to fix the quantifier for types $\left(\forall_{\text {types }}\right)$ to appear only on the very top position in formulas.

$$
\begin{aligned}
& \frac{\gamma_{\mathcal{C}}^{\mathcal{T}} \vdash s: \tau \quad \gamma_{\mathcal{C}}^{\mathcal{T}} \vdash t: \tau}{\gamma_{\mathcal{C}}^{\mathcal{T}} \vdash s \approx t: o_{\mathcal{B}}}(\approx) \quad \frac{\gamma_{\mathcal{C}}^{\mathcal{T}} \vdash \phi_{1}: o_{\mathcal{B}} \quad \gamma_{\mathcal{C}}^{\mathcal{T}} \vdash \phi_{2}: o_{\mathcal{B}}}{\gamma_{\mathcal{C}}^{\mathcal{T}} \vdash \phi_{1} \# \phi_{2}: o_{\mathcal{B}}}(\# \in\{\wedge, \vee, \Rightarrow, \Leftrightarrow\}) \quad \frac{\gamma_{\mathcal{C}}^{\mathcal{T}} \vdash \phi: o_{\mathcal{B}}}{\gamma_{\mathcal{C}}^{\mathcal{T}} \vdash \neg \phi: o_{\mathcal{B}}}(\neg) \\
& \frac{\mathrm{p}: \forall \alpha_{1}: \mathrm{C}_{1} \ldots \alpha_{\mathrm{n}}: \mathrm{C}_{\mathrm{n}} \cdot \tau_{1} \ldots \tau_{\mathrm{m}} \rightarrow \mathrm{o}_{\mathcal{B}} \in \mathcal{P} \quad \gamma_{\mathcal{C}}^{\mathcal{T}} \vdash t_{1}: \tau_{1} \sigma \quad \ldots \quad \gamma_{\mathcal{C}}^{\mathcal{T}} \vdash t_{n}: \tau_{n} \sigma}{\gamma_{\mathcal{C}}^{\mathcal{T}} \vdash p<\alpha_{1} \sigma, \ldots, \alpha_{m} \sigma>\left(t_{1}, \ldots, t_{n}\right): o_{\mathcal{B}}} \text { (predicate) }
\end{aligned}
$$

and for all $i: 1 . . m . \mathcal{C} \vdash \alpha_{i} \sigma: C_{i}$

$$
\frac{\gamma_{\mathcal{C}}^{\mathcal{T}[u \mapsto \tau]} \vdash \phi: o_{\mathcal{B}}}{\gamma_{\mathcal{C}}^{\mathcal{T}} \vdash \# u: \tau . \phi: o_{\mathcal{B}}}(\# \in\{\forall, \exists\}) \quad \frac{\gamma_{\mathcal{C}}^{\mathcal{T}} \vdash \phi: o_{\mathcal{B}}}{\gamma_{\mathcal{C}}^{\mathcal{T}} \vdash \phi: o}\left({ }_{\text {top }}\right) \quad \frac{\gamma_{\mathcal{C}[\alpha \mapsto C]}^{\mathcal{T}} \vdash \phi: o}{\gamma_{\mathcal{C}}^{\mathcal{T}} \vdash \forall_{\text {types }} \alpha: C . \phi: o}\left(\forall_{\text {types }}\right)
$$

We call a formula $\phi$ well-typed if and only if one can derive starting from

$$
\gamma_{\mathcal{C}}^{\mathcal{T}} \vdash \phi: o
$$

a proof tree by applying the above rules with an empty $\gamma_{\mathcal{C}}^{\mathcal{T}}$. 


\section{Semantic}

Structure Given a polymorphic+typeclass first-order language signature the polymorphic+typeclass first-order language structure is a tuple $\Sigma=(\mathcal{U}, \mathcal{D}, \mathcal{I})$

$\mathcal{U}$ The (non-empty) universe.

$\mathcal{D}$ The (non-empty) domains representing (non-empty) types (subsets of $\mathcal{U}$ ).

$\mathcal{I}$ The interpretation function

We denote the interpretation of a well typed \{formula,term,type,typeclass\} $\mathrm{t}$ as $\llbracket t \rrbracket_{\theta}^{\mathcal{I}},{ }^{1}$ Where $\theta$ is a mapping from type variables to elements of $\mathcal{D}$ and $\xi$ is a mapping from term variables to elements of $\mathcal{U}$. We write $\theta[\alpha \mapsto \tau]$ (respectively $\xi[u \mapsto e]$ ) to represent a new mapping which is identical to $\theta(\xi)$ but maps $\alpha$ to $\tau$ ( $u$ to $e$ ). To interpret a (non-sub) formula, $\theta$ and $\xi$ are set to be empty.

\begin{tabular}{|c|c|c|}
\hline Types & $\begin{array}{l}\llbracket \alpha \rrbracket_{\theta}^{\mathcal{I}} \\
\llbracket \kappa\left(\tau_{1}, \ldots, \tau_{n}\right) \rrbracket_{\theta}^{\mathcal{I}}\end{array}$ & $\begin{array}{l}::=\theta(\alpha) \\
::=\quad \kappa^{\mathcal{I}}\left(\llbracket \tau_{1} \rrbracket_{\theta}^{\mathcal{I}}, \ldots, \llbracket \tau_{n} \rrbracket_{\theta}^{\mathcal{I}}\right)\end{array}$ \\
\hline Term & $\begin{array}{l}\llbracket u \rrbracket_{\theta, \xi}^{\mathcal{I}} \\
\llbracket f<\tau_{1}, \ldots, \tau_{n}>\left(t_{1}, \ldots, t_{m}\right) \rrbracket_{\theta, \xi}^{\mathcal{I}}\end{array}$ & $\begin{array}{l}::=\quad \xi(u) \\
::= \\
f^{\mathcal{I}}<\llbracket \tau_{1} \rrbracket_{\theta}^{\mathcal{I}}, \ldots, \llbracket \tau_{n} \rrbracket_{\theta}^{\mathcal{I}}>\left(\llbracket t_{1} \rrbracket_{\theta, \xi}^{\mathcal{I}}, \ldots, \llbracket t_{m} \rrbracket_{\theta, \xi}^{\mathcal{I}}\right)\end{array}$ \\
\hline Formulas & $\begin{array}{l}\llbracket p<\tau_{1}, \ldots, \tau_{n}>\left(t_{1}, \ldots, t_{m}\right) \rrbracket_{\theta, \xi}^{\mathcal{I}} \\
\llbracket s \approx t \rrbracket_{\theta, \xi}^{\mathcal{I}} \\
\llbracket s \wedge t \rrbracket_{\theta, \xi}^{\mathcal{I}} \\
\llbracket s \vee t \rrbracket_{\theta, \xi}^{\mathcal{I}} \\
\llbracket s \Rightarrow t \rrbracket_{\theta, \xi}^{\mathcal{I}} \\
\llbracket s \Leftrightarrow t \rrbracket_{\theta, \xi}^{\mathcal{I}} \\
\llbracket \neg t \rrbracket_{\theta, \xi}^{\mathcal{I}} \\
\llbracket \forall u: \tau \cdot \psi \rrbracket_{\theta, \xi}^{\mathcal{I}} \\
\llbracket \exists u: \tau \cdot \psi \rrbracket_{\theta, \xi}^{\mathcal{I}} \\
\llbracket \forall_{\text {types }} \alpha: C \cdot \psi \rrbracket_{\theta, \xi}^{\mathcal{I}}\end{array}$ & 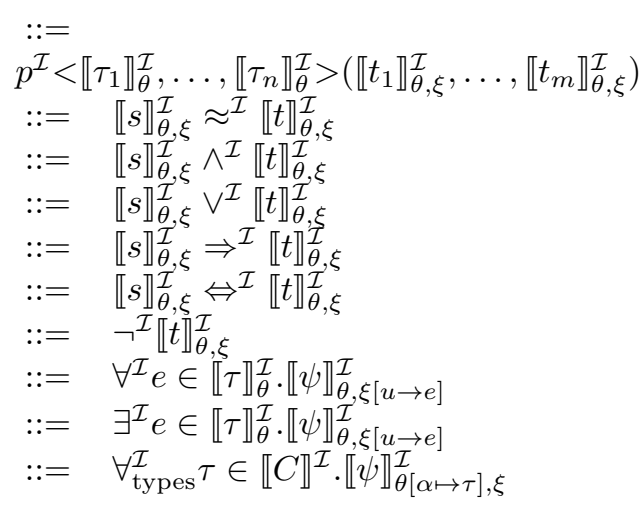 \\
\hline Type Class & $\begin{array}{l}\llbracket \emptyset \rrbracket^{\mathcal{I}} \\
\llbracket C \rrbracket^{\mathcal{I}} \\
\llbracket c \rrbracket^{\mathcal{I}}\end{array}$ & $\begin{array}{l}::=\mathcal{D} \\
::=\bigcap_{c \in C} \llbracket c \rrbracket^{\mathcal{I}} \\
::=\text { least fix point of rules } 1 \text { and } 2\end{array}$ \\
\hline
\end{tabular}

\section{Type Class Fix point}

1. For all declarations $\forall \alpha_{1}: C_{1}, \ldots, \alpha_{n}: C_{n} . \tau: c \in \mathcal{T}$ require that all valid instantiations of $\tau$ 's type variables are also of $\tau$ 's type class $c$ :

$$
\left(\bigwedge_{1 \leq i \leq n} \tau_{i} \in \llbracket C_{i} \rrbracket^{\mathcal{I}}\right) \Rightarrow\left(\ldots, \alpha_{i} \mapsto \tau_{i}, \ldots\right)(\tau) \in \llbracket c \rrbracket^{\mathcal{I}}
$$

2. For all declarations $c_{1} \subseteq c_{2} \in \mathcal{T C}$ require that if a type term $\tau$ is element of the subclass $c_{1}$ then $\tau$ is also element of the superclass $c_{2}$.

\footnotetext{
${ }^{1} \xi$ is not needed for interpretation of types. $\theta, \xi$ are not needed for interpretation of type classes.
} 


$$
\tau \in \llbracket c_{1} \rrbracket^{\mathcal{I}} \Rightarrow \tau \in \llbracket c_{2} \rrbracket^{\mathcal{I}}
$$

Following from Tarski's fix point theorem the rules 1 and 2 have a least fix point.

\section{Typed Superposition}

Preprocessing Initially formulas are transformed into Clause Normal Form 2] (CNF). This is unaffected by the type system, except for skolemization. Skolemization has to add corresponding type declarations into $\mathcal{T}$. The necessary declarations are obvious, the existential variables' type becomes the Skolem function's return type and its argument types are the types of the universal variables, whose scope includes the existential variable.

Since we only have universally quantified variables in CNF, we omit all quantifiers and annotate variables $(u)$ with their type $(\tau)$ and write $u\{\tau\}$ to express that $u$ is of type $\tau$. Functions and predicates ${ }^{2}$ are represented by their return type and their argument terms and 'true' types arguments. In detail:

is represented by

$$
f<\tau_{\alpha_{1}}, \ldots, \tau_{\alpha_{n}}>\left(t_{1}, \ldots, t_{m}\right)
$$

$$
f\left\{\tau_{r}, \tau_{\alpha_{i}}, \ldots\right\}\left(t_{1}, \ldots, t_{m}\right)
$$

where $\forall \alpha_{1}: C_{1} \ldots \alpha_{n}: C_{n} \cdot \tau_{1} \ldots \tau_{m} \rightarrow \tau \in \mathcal{F}$ is f's declaration. $\tau_{r}$ is defined to be $\tau$ instantiated by the substitution $\sigma$ such that for all $j$ in $1 . . n$ : $\alpha_{j} \sigma$ is $\tau_{a_{j}}$ and for all $\mathrm{j}$ in $1 . . m$ : the type of $t_{j}$ is $\tau_{j} \sigma$. The $\tau_{\alpha_{i}}$ are of those $i$ whose $\alpha_{i}$ does not occur in $\tau$ and not in any $\mathrm{j}$ in $1 . . m$ : $\tau_{j}$ (and are ordered increasing in $i$ ). This is useful to e.g. have polymorphic unary predicates. Clearly both representation, together with $\mathcal{F}$ or $\mathcal{P}$, provide the same information.

The advantage of the second form is that it requires no lookup of $\mathcal{F}$ and $\mathcal{P}$ to perform unification between variables and functions.

Substitutions Let $\sigma$ be a mapping $X \rightarrow T_{\Sigma}(X)$ which maps term variables to terms and type variables to type terms. We write substitutions as $\left(u_{1} \mapsto t_{1}, \ldots, \alpha_{1} \mapsto \tau_{1} \ldots\right)$ and instead of $\sigma(x)$ we also write $x \sigma$. We define $\sigma\left[u_{1} \mapsto t_{1}\right]$ to be $t_{1}$ for $u_{1}$ and for all other variables $u$ to be $u \sigma$ (correspondingly for $\alpha$ ). We define the application of $\sigma$ on terms as follows:

and on type terms as:

$$
f\{\tau\}\left(t_{1}, \ldots, t_{m}\right) \sigma::=f\{\tau \sigma\}\left(t_{1} \sigma, \ldots, t_{m} \sigma\right)
$$

$$
\kappa\left(\tau_{1}, \ldots, \tau_{n}\right) \sigma::=\kappa\left(\tau_{1} \sigma, \ldots, \tau_{n} \sigma\right)
$$

We call a substitution $\sigma$ grounding for a given term $\mathrm{t}$ if $\mathrm{t} \sigma$ contains no variables; a unifier of the terms $t_{1}$ and $t_{2}$ if $t_{1} \sigma \approx t_{2} \sigma$; more general than $\sigma_{2}$ if for all terms $t$ there exists $\sigma_{1}$ such that $t \sigma \sigma_{1} \approx t \sigma_{2}$ and a most general unifier if it is more general than all other unifiers.

Unification We now give the unification rules for terms; starting with non-variable terms: Note that preprocessing has simplified unification for functions and predicates.

$$
\begin{array}{llll}
1 & f\left\{\tau_{l}\right\}\left(t_{l_{1}}, \ldots, t_{l_{m}}\right) \doteq f\left\{\tau_{r}\right\}\left(t_{r_{1}}, \ldots, t_{r_{m}}\right), \mathrm{E} & \Rightarrow & \tau_{l} \doteq \tau_{r}, t_{l_{1}} \doteq t_{r_{1}}, \ldots, t_{l_{m}} \doteq t_{r_{m}}, \mathrm{E} \\
2 & p\left(t_{l_{1}}, \ldots, t_{l_{m}}\right) \doteq p\left(t_{r_{1}}, \ldots, t_{r_{m}}\right), \mathrm{E} & \Rightarrow & t_{l_{1}} \doteq t_{r_{1}}, \ldots, t_{l_{m}} \doteq t_{r_{m}}, \mathrm{E} \\
3 & \kappa\left(\tau_{1_{l}}, \ldots, \tau_{n_{l}}\right) \doteq \kappa\left(\tau_{1_{r}}, \ldots, \tau_{n_{r}}\right), \mathrm{E} & \Rightarrow & \tau_{l} \doteq \tau_{l_{1}} \doteq \tau_{r_{1}}, \ldots, t_{l_{n}} \doteq t_{r_{n}}, \mathrm{E}
\end{array}
$$

for term variables:

\footnotetext{
${ }^{2}$ We use the same transformation for predicates, but omit the return type.
} 


$$
\begin{array}{lllll}
4 & t \doteq u, \mathrm{E} & \Rightarrow & =t, \mathrm{E} & \\
5 & u\left\{\tau_{u}\right\} \doteq t\left\{\tau_{t}\right\}, \mathrm{E} & \Rightarrow & u\left\{\tau_{u}\right\}=t\left\{\tau_{t}\right\}, \tau_{u} \doteq \tau_{t},\left(u\left\{\tau_{u}\right\} \mapsto t\left\{\tau_{t}\right\}\right)(\mathrm{E}) & \text { t non-variable } \\
6 & u_{l}\left\{\tau_{l}\right\} \doteq u_{r}\left\{\tau_{r}\right\}, \mathrm{E} & \Rightarrow & u_{l}\left\{\tau_{l}\right\}=u_{r}\left\{\tau_{r}\right\}, \tau_{l} \doteq \tau_{r},\left(u_{l}\left\{\tau_{l}\right\} \mapsto u_{r}\left\{\tau_{r}\right\}\right)(\mathrm{E}) &
\end{array}
$$

for type variables:

$$
\begin{array}{rrr}
7 \alpha_{1}\left\{C_{1}\right\} \doteq \alpha_{2}\left\{C_{2}\right\}, \mathrm{E} \Rightarrow \quad \begin{array}{l}
C_{3}=C_{1} \cup C_{2}, \alpha_{1}\left\{C_{1}\right\}=\alpha_{3}\left\{C_{3}\right\}, \alpha_{2}\left\{C_{1}\right\}=\alpha_{3}\left\{C_{3}\right\}, \\
\left(\alpha_{1}\left\{C_{1}\right\} \mapsto \alpha_{3}\left\{C_{3}\right\}, \alpha_{2}\left\{C_{1}\right\} \mapsto \alpha_{3}\left\{C_{3}\right\}\right)(E)
\end{array} \\
\alpha_{1}\{C\} \doteq \tau, \mathrm{E} \Rightarrow \quad \alpha_{1}\{C\}=\tau \sigma,\left(\sigma\left[\alpha_{1}\{C\} \mapsto \tau \sigma\right]\right)(\mathrm{E}) \quad \begin{array}{c}
\alpha_{3} \text { fresh and } C_{3} \text { pop. } \\
\sigma \operatorname{mgs}_{\tau, C}
\end{array}
\end{array}
$$

Where $\operatorname{vars}(t)$ is the set of all variables that are $t$ or subterms of $t$. A variable is fresh if it was not previously used anywhere. $C$ pop. tests if $C$ is populated, meaning that it has at least one type constructor which can construct all type classes $c \in C$. A substitution $\sigma$ is the most general substitution $\left(\mathbf{m g s}_{\tau, C}\right)$ of $\tau$ and $C$ if for all $\sigma_{2}$ exists a $\sigma_{3}$ such that if $\tau \sigma_{2}$ is a member of $C$ (according to $\mathcal{T}$ ) then $\sigma_{2}=\sigma \sigma_{3}$. This has no effect on ground type terms and will only restrict type variables' type classes constraint, since restricting a type variable is more general than instantiating it ${ }^{3}$

Two terms $t$ and $s$ are unifiable if and only if $t \doteq s$ can be transformed by the above rules into a set of equations not containing $\doteq$. The most general unifier ${ }^{4}$ can be derived from the resulting equations.

Typed Knuth-Bendix Order The Knuth-Bendix Order (KBO) is a widely used order in superposition based provers. Based on the presentation in [1, p. 124] we extend the standard KBO to our typed setting, we claim that it is still a simplification order:

Let $w_{f}$ be a function that maps function symbols to positive numbers, $w$ its extension to terms and $>_{f}$ be an ordering on function symbols. We define $\mathrm{s} \succ_{K B O}$ t to hold if and only if:

1. For all term variables $\mathrm{u}:|s|_{u} \geq|t|_{u}$ and $w(s)>w(t)$, or

2. For all term variables $\mathrm{u}:|s|_{u} \geq|t|_{u}$ and $w(s)=w(t)$, and one of the following:

(a) There exists a unary function symbol $f$, a variable $u$ and a positive integer $n$ such that $s=(f\{\tau\})^{n}(u)$ and $t=u$

(b) There exists functions symbols $f, g$ such that $f>_{f} g$ and $s=f\{\tau\}\left(s_{1}, \ldots, s_{n}\right)$ and $t=g\{\tau\}\left(t_{1}, \ldots, t_{n}\right)$

(c) There exists a function symbol $f$ and an index $i, 1 \leq i \leq n$, such that $s=$ $f\{\tau\}\left(s_{1}, \ldots, s_{n}\right), t=f\{\tau\}\left(t_{1}, \ldots, t_{n}\right)$ and $s_{1}={ }_{T e r m} t_{1}, \ldots, s_{i-1}=_{\text {Term }} t_{i-1}$ and $s_{i} \succ_{K B O} t_{i}$

(d) $s \succ_{\tau} t$

And the following definitions:

- $\tau_{s} \succ_{\tau} \tau_{t}$ iff there exists a $\sigma$ such that $\tau_{s} \sigma \neq \tau_{s}$ and $\tau_{s} \sigma=\tau_{t}$

- $s \succ_{\tau} t$ iff there exists a function symbol f such that $s=f\left\{\tau_{s}\right\}\left(s_{1}, \ldots, s_{n}\right)$,

and

$$
t=f\left\{\tau_{t}\right\}\left(t_{1}, \ldots, t_{n}\right), \quad s_{1}={ }_{\text {Term }} t_{1}, \ldots, s_{n}==_{\text {Term }} t_{n}, \quad s_{1} \succeq_{\tau} t_{1}, \ldots, s_{n} \succeq_{\tau} t_{n}
$$

$$
\tau_{s} \succ_{\tau} \tau_{t} \text { or }\left(\tau_{s}=\tau_{t} \text { and there exists an index } \mathrm{i}, 1 \leq \mathrm{i} \leq \mathrm{m} \text {, such that } s_{i} \succ_{\tau} t_{i}\right)
$$

\footnotetext{
${ }^{3}$ Except in the corner case, where restricting the type variable and instantiating it, represents the same type terms.

${ }^{4}$ The most general unifier is unique, which we will not show here.
} 
- $=_{T e r m}$ to be equality but only considering non-type (sub)terms.

In its presented form, $\succ_{K B O}$ allows ordering of terms with same type and additionally if the non-type terms are equal, more generally typed terms are smaller than stricter typed terms. One could further relax 2a, 2b and 2c to more general types, similar to $\succ_{\tau}$, but might have to also consider subterms' type terms.

Inferences' Side Conditions The following side conditions are used in the inference rules below. Let $\prec$ be a fixed simplification order 1 .

1. $\sigma$ is the most general unifier of $s$ and $s_{2}$

2. $\sigma$ is the most general unifier of $s$ and $s^{\prime}$

3. $s_{2}$ is not a term variable

4. $t \sigma \npreceq t^{\prime} \sigma$

5. $s \sigma \npreceq s^{\prime} \sigma$

6. $\left(t \approx t^{\prime}\right) \sigma$ strictly maximal in $\left(D^{\prime} \vee t \approx t^{\prime}\right) \sigma$, nothing selected

7. $\left(s \approx s^{\prime}\right) \sigma$ strictly maximal in $\left(C^{\prime} \vee s \approx s^{\prime}\right) \sigma$, nothing selected

8. $\left(\left(s \not s^{\prime}\right) \sigma\right.$ maximal in $\left(C^{\prime} \vee s \approx s^{\prime}\right) \sigma$, nothing selected $) \vee s \not \approx s^{\prime}$ selected

9. $D^{\prime} \vee t \approx t^{\prime} \nsucceq C^{\prime} \vee s\left[s_{2}\right]_{p} \ldots s^{\prime}$

10. $(s \approx t) \sigma$ maximal in $\left(C^{\prime} \vee s^{\prime} \approx t^{\prime} \vee s \approx t\right) \sigma$, nothing selected

Inferences We take the superposition calculus of [3] and sketch that it still refutationally complete in our typed setting.

1. Positive Superposition (PSup) with side conditions 1, 3, 4, 5, 6, 7, and 9

$$
\frac{D^{\prime} \vee t \approx t^{\prime} \quad C^{\prime} \vee s\left[s_{2}\right]_{p} \approx s^{\prime}}{\left(D^{\prime} \vee C^{\prime} \vee s\left[t^{\prime}\right]_{p} \approx s^{\prime}\right) \sigma}(\text { PSup })
$$

2. Negative Superposition (NSup) with side conditions 1, 3, 4, 5, 6, 8 and 9

$$
\frac{D^{\prime} \vee t \approx t^{\prime} \quad C^{\prime} \vee s\left[s_{2}\right]_{p} \not \approx s^{\prime}}{\left(D^{\prime} \vee C^{\prime} \vee s\left[t^{\prime}\right]_{p} \not \approx s^{\prime}\right) \sigma}(\text { Sup })
$$

3. Equality Resolution (ER) with side conditions 2 and 8

$$
\frac{C^{\prime} \vee s \not \varpi s^{\prime}}{C^{\prime} \sigma}(E R)
$$

4. Equality Factoring (EF) with side conditions $2,10 s^{\prime} \sigma \npreceq t^{\prime} \sigma$ and $s \sigma \npreceq t \sigma$,

$$
\frac{C^{\prime} \vee s^{\prime} \approx t^{\prime} \vee s \approx t}{\left(C^{\prime} \vee s^{\prime} \approx t^{\prime} \vee t \not t^{\prime}\right) \sigma}(E F)
$$


Refutational Completeness Sketch In order to show refutational completeness of typed superposition, two main theorems have to be proven. First the inference rules have to be shown well-typedness preserving. This is already sufficient to prove the ground version refutational complete. Then new versions of the lifting lemmas have to be shown. The remaining completeness proof is essentially unchanged.

Lemma 1 Well typedness is determined only by the well typedness of the argument and the typing rule of the construct.

Lemma 2 For all well typed terms $t_{1}, t_{2}, t_{3}$ and substitutions $\sigma$, if $\sigma$ is the most general unifier of $t_{2}$ and $t_{3}$ then $t_{1} \sigma$ is well typed.

Lemma 3 If $t \approx t^{\prime}$ then the type of $t$ and $t^{\prime}$ is identical.

Lemma 4 For each inference if the premises are well typed so is the conclusion.

Proof Sketch

Equality Resolution : By Lemma 2

Equality Factoring : By Lemma $3 s, t \sigma$ and $t^{\prime} \sigma$ all have the common type $\tau$.

Thus the equations from the conclusion are also well-typed.

$C^{\prime} \sigma$ is well typed by Lemma 2 .

Superposition $\quad:$ By Lemma $3 t \sigma$ and $t^{\prime} \sigma$ have the common type $\tau$.

Thus by Lemma 1 they can be used interchangeably.

$C^{\prime} \sigma$ and $D^{\prime} \sigma$ are well typed by Lemma 2 .

Lemma 5 If there exists a $\sigma$ such that $t_{1} \sigma \approx t_{2} \sigma$ then there exists a (unique up to renaming) most general unifier.

The completeness of typed ground superposition now follows from the completeness proof for untyped superposition given in [3]. To show the lifting, we need to show for each inference that if $\sigma$ is a grounding substitution, then every inference from $C \sigma^{5}$ is an ground instance of an inference of $\mathrm{C}$. After that the model construction lemma and the remaining completeness proof can be reused without further changes.

Redundancy Criteria Since we reused most of the original completeness proof also the redundancy criteria holds. Thus all commonly used reductions can be also used in the typed superposition calculus.

\section{Implementation}

We have implemented a prototype of the above calculus from scratch in Scala. Unification is implemented with the help of unification contexts, with separate contexts for term variables and type variables. Additionally we implemented several of the reductions described in [14; namely we have implemented Subsumption Deletion, Trivial Literal Elimination, Condensation, Unit Rewriting and Assignment Equality Deletion, as well as simple forms of Merging Replacement Resolution (MRR) and Tautology Elimination. We implement sharing, by keeping term and type variable numbers separate (and normalizing them), the number of distinct type terms is usually low. We use a worked-off/usable style main loop and fully reduce both sets. Indexing is implemented as a combination of a naive implementation of Substitution Trees[1] and Feature Vector Indexing[13].

Overall the implementation is reasonable, but not expected to be remotely competitive to an efficient $\mathrm{C}$ implementation featuring more advanced reduction techniques, such as full MRR

\footnotetext{
${ }^{5}$ And $D \sigma$ for superposition, where $C$ and $D$ do not share variables
} 
and Splitting. A web interface is available from the abstract's web page.

\subsection{Specifics of the Polymorphic+Typeclass implementation}

In this section we describe the implementation necessary to specifically support Polymorphic+Typeclass superposition. We were able to keep the implementation of the inferences and reductions completely without any type-system related code, since the generic interfaces for indexing, unification and substitution take care of the type-system handling.

Start up We have extended the syntax, where the changes to the untyped settings are restricted to the additional information to be parsed. We also save all declarations, which are used for type-class unification and for assertions that ensure well-typedness throughout our prototype. CNF generation is also mostly unchanged. Only skolemization has to create adequate type declarations, which can be easily derived from the generated term.

Datastructures We use algebraic datastructures to encode literals, terms and type terms in the processed form described in the beginning of section 6. Clauses are unchanged by the type-system.

Assertions Our prototype is a relatively new piece of software which we use to experiment with. To ensure that the implementation does what we expect from it we employ Scala's assertions. We employ over 300 distinct assertions, checking various properties such as: Well typed-ness, correctness of unifiers, assumptions on term sharing. We also ensure more complex properties such as that clauses are not lost from our Worked Off / Usable sets by tracking all reductions and inferences and tracing them to the input clause set.

Sharing Sharing can be easily extended to also share type-terms. We use a hash map based sharing and literals, terms and type terms are are only created by the sharing.

Indexing For indexing we treat typed terms as untyped terms, the indexing "pretends" that type terms are just normal first-order terms. This yields all valid unifications and some unifications which are not well-typed, in particular since the indexing ignores type-classes. Therefore all unifications are verified before they are used. For Feature Vector indexing, the original untyped variant can also be used, and types can be treated as an additional feature. We have not noticed any further changes to be required. We are thus able to reuse existing datastructures almost unchanged, by adding post indexing unification checks, which verify the well-typedness.

Unification Unification is where most of the type-system related changes can be contained to. It is a straight forward implementation of the previously described unification algorithm. We have also implemented it to check/generate instantiations and renamings.

\section{Evaluation}

Setup We have evaluated our prototype on problems generated by Sledgehammer 4 from Isabelle/HOL theories, of areas as diverse as the fundamental theorem of algebra, the com- 
pleteness of Hoare logic and the type soundness of a subset of Java ${ }^{6}$. The 5797 problems were tested on 4 different type encodings, at 4 different fact sizes (16, 64, 256 and 1024 axioms) with 2 hours of runtime on one HyperThread of an 2xIntel Xeon E5620 with a JVM heap size of 1 GB.

\section{The type encodings are:}

Poly Tags Encoding polymorphism into untyped first-order with the help of special typing function symbols (see [5, p. 9]). According to [7, p. 11,12] this is a more efficient encoding than guard predicates for polymorphism.

Monomorph An incomplete encoding of polymorphism by picking relevant monomorphic instances (see [9]). According to [7, p. 11,12] currently the most efficient encoding.

Poly Native Using native polymorphism but encoding type classes as predicates.

Type Classes Using native polymorphism with its type classes.

Comparison to SPASS For reference we have compared the performance of our prototype at 256 axioms and Mono and Poly Tags encodings with the performance of a SPASS version specifically optimized for Isabelle/HOL[7]. SPASS is significantly faster than our prototype. Our tool can derive $75 \%$ (208 vs. 277) of SPASS's Monomorph proof count and 74\% (162 vs. 219) of its Poly Tags proof count. Since our tool exhibits similar relative performance on the encodings that SPASS supports, we believe that our results will also carry over to efficient implementations.

Impact of the axioms The fewer axioms a problem has the easier it is, since fewer axioms mean fewer clauses and thus fewer possible inferences. It is often possible to quickly find saturations for 16 axioms, but seldom for the bigger axiom sizes. The more axioms a problem has the more likely it is that the relevant axioms for the proof are included. These observations are usually exploited by running a portfolio of different settings (slices). E.g. as the first slice with 16 axioms and a short timeout, followed by a larger axiom set since with longer timeout. Our evaluation will focus on a single slice, because here we are only interested in how well the different type encodings perform. In particular how well a type encoding handles additional axioms, e.g. how well it scales.

Scalability of type encodings Figure 1 shows the success rate and scalability of the tested encodings. Where the $\mathrm{X}$ axis denotes the number of axioms, and the $\mathrm{Y}$ axis the percentage of problems proved, in a single run of up to 2 hours. For all encodings, the most successful axiom set size tested is 256 axioms. The Poly Tags encoding performs worst. For all axiom sizes it solves fewer problems then the native encodings. Its performance also deteriorates the most when increasing the axiom set from 256 axioms to 1024, resulting in a drop of the success rate of 8 percentage points. The Monomorph and the Type Classes encoding both only suffer a drop of 3 percentage points.

\footnotetext{
${ }^{6}$ We evaluated on Arr, FFT, FTA, Hoa, Jin, Lam and NS see 4 for details

${ }^{7}$ Our original set had 694 problems, but 115 had to be excluded because they were missing typing information.
} 


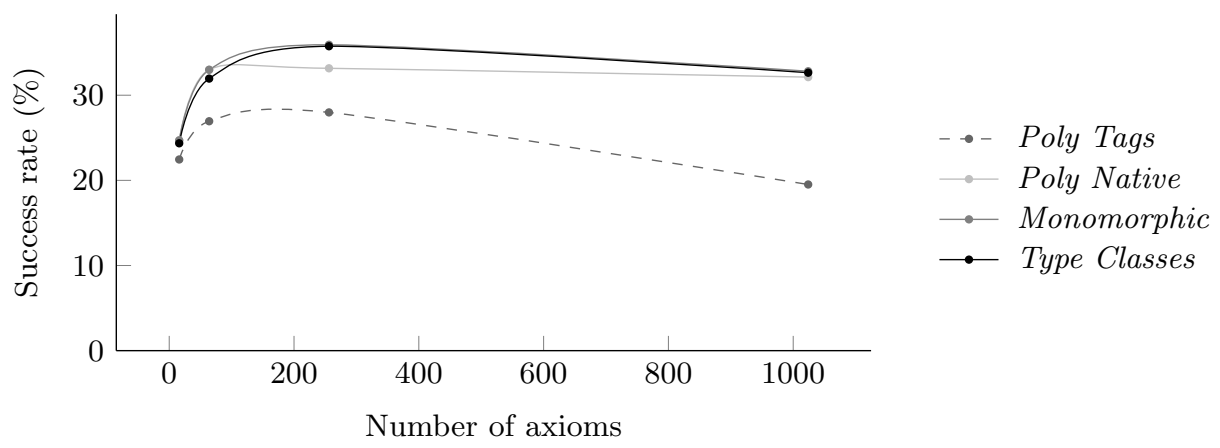

Figure 1: Success rate and scalability of each type encoding

Overall the Monomorph encoding performs best. It is closely followed by the Type Classes encoding which at worst (64 axioms) solves $96.8 \%$ of the problems that Monomorph solves. Type Classes and Monomorph are almost identical on the other axiom set sizes.

Speed For small problems the Type Classes encoding is slower than the Monomorph encoding. This can also be seen in figure 4, two scatter plots in which the logarithmic Monomorph runtime is on the $\mathrm{Y}$-axis and the logarithmic Type Classes runtime is on the $\mathrm{X}$-axis. For 16 axioms (left) almost all results are in the lower right half, signifying that Monomorph is faster: a runtime of $\varnothing 3.7 \mathrm{~s}$ versus $\varnothing 2.0$ s (on 137 problems both solve).

\subsection{Detailed Evaluation}

As can be seen in Fig. 1 the most successful axiom set size, for all encodings, is 256 axioms. Thus we will now focus on this to provide a more detailed evaluation of the encoding. The following table provides the number of proofs an the average time it took to find them:

\begin{tabular}{l|l|l} 
Encoding & Proofs & Average Time \\
\hline Monomorph & 208 & $384 \mathrm{~s}$ \\
\hline Type Classes & 207 & $137 \mathrm{~s}$ \\
\hline Poly Native & 192 & $133 \mathrm{~s}$ \\
\hline Poly Tags & 162 & $115 \mathrm{~s}$
\end{tabular}

Poly Tags has the fewest proofs and even though it has the lowest average runtime it is actually the slowest if we compare it to the other encodings only on the problems both solve. Its solutions overlap with Type classes on 154 proofs. On those Poly Tags' average runtime is $\varnothing 119 \mathrm{~s}$, whereas Type Classes' average runtime is only $\varnothing 20 \mathrm{~s}$. The other encodings are also faster, the overlap with Monomorph is also 154 proofs, where Poly Tags' average runtime is $\varnothing 118$ s and Monomorph's average runtime is $\varnothing 80 \mathrm{~s}$. The overlap with Monomorph is also 154 proofs, where Poly Tags' average runtime is $\varnothing 108 \mathrm{~s}$ and Poly Native's average runtime is $\varnothing 29 \mathrm{~s}$.

Below we show a scatter plot comparing the runtime of Poly Tags and Type Classes. The $\mathrm{X}$ axis is the logarithmic runtime of Type Classes and the Y axis the logarithmic runtime of Poly Tags. The results cluster in the upper-left half, again showing that Type Classes is faster.

Poly Native has similarly to Poly Tags fewer proofs and a lower average runtime than Type Classes and Monomorph. Poly Native is actually faster, but less successful. Its solutions overlap 


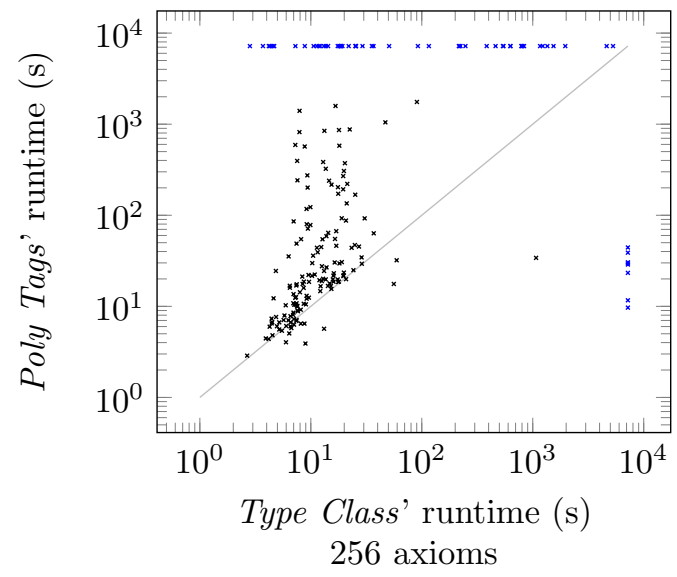

Figure 2: Runtime of Poly Tags encoding versus native Type Classes.

with Type classes on 184 proofs. On those Poly Native's average runtime is $\varnothing 78 \mathrm{~s}$, whereas Type Classes' average runtime is $\varnothing 122 \mathrm{~s}$. The overlap with Monomorph is on 179 proofs, where Poly Native's average runtime is $\varnothing 102 \mathrm{~s}$ and Monomorph's average runtime is $\varnothing 239 \mathrm{~s}$. Below we show again a scatter plot, comparing the runtime of Poly Native and Type Classes. Note that the average runtime is dominated by the long running proofs in the upper right corner. For the faster proofs (up to 100s runtime) Type Classes is actually slightly faster. We believe that this shows that on some problems the possible advantages of native type classes do not compensate their overhead during unification. The advantages start to show on the larger (e.g. 1024) axiom sizes. Here Type Classes' average runtime is $\varnothing 195 \mathrm{~s}$ and Poly Native's average runtime is $\varnothing 248 \mathrm{~s}$ on 193 proofs they both find.
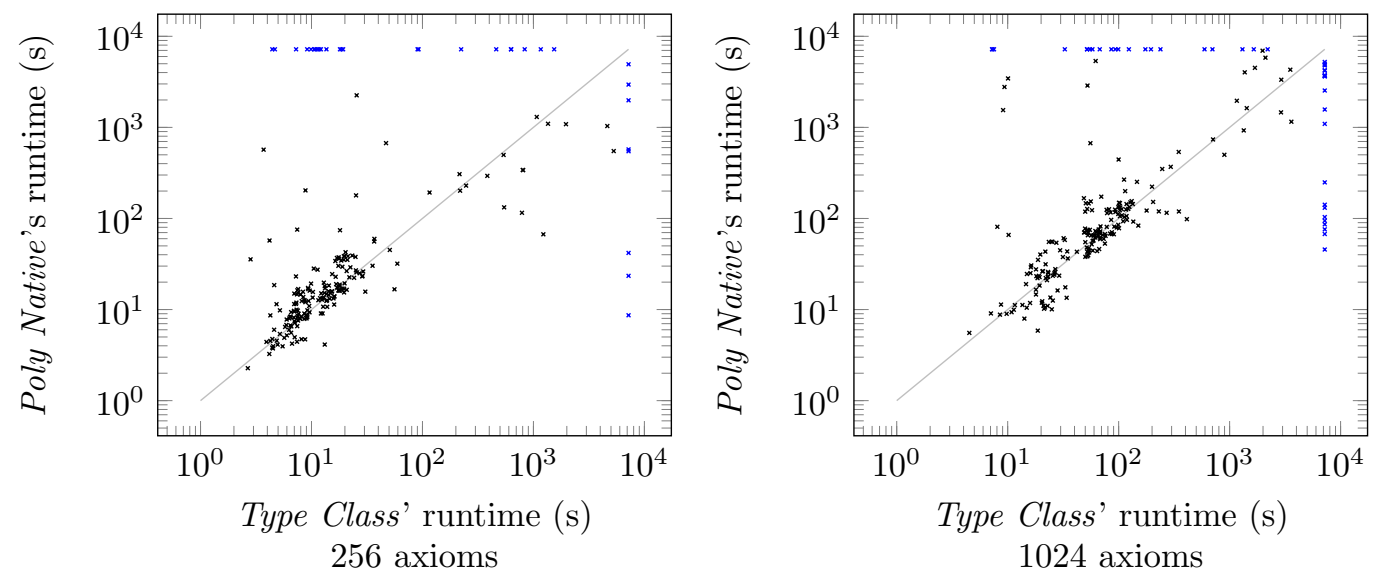

Figure 3: Runtime of Poly Native encoding versus native Type Classes with two different axiom set sizes, on the left with 256 axioms and on the right with 1024 axioms. 
Monomorph and Type Classes find a similar amount of proofs with Type Classes having a lower average total runtime. They overlap on 193 proofs. On those Type Classes has an average runtime of $\varnothing 85 \mathrm{~s}$ and Monomorph an average runtime of $\varnothing 198 \mathrm{~s}$. It is also faster on axiom set sizes of 1024, but initially it is not. For an axiom set size of 16, Monomorph is almost uniformly faster. The overlap for 16 axioms is 140 proofs, where Monomorph has an average runtime of $\varnothing 2 \mathrm{~s}$ and Type Classes of $\varnothing 4 \mathrm{~s}$. We believe that in an efficient implementation both most of the runtimes would be well below 1s (for a size of 16 axioms).
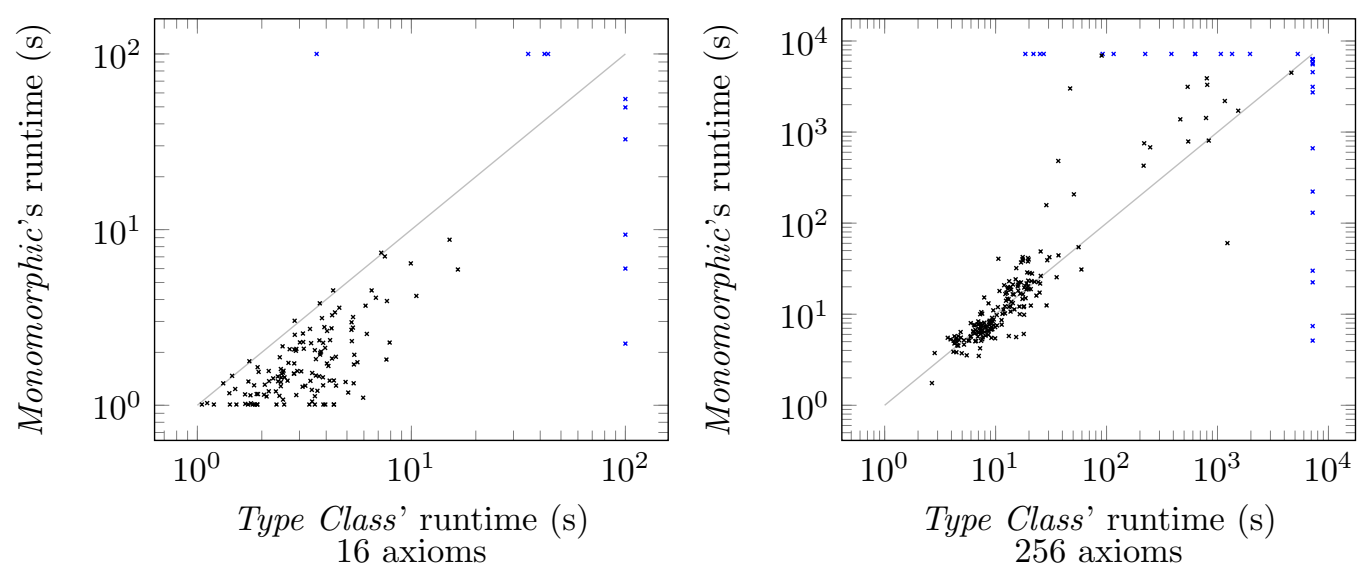

Figure 4: Runtime of Monomoprh encoding versus native Type Classes with two different axiom set sizes, on the left with 16 axioms and on the right with 256 axioms.

\section{Conclusion}

We have presented a typed form of superposition with rank-1 polymorphism and type classes. We have given its semantic; adapted substitution, unification and the commonly used KBO ordering to our new setting. We have also presented an initial preprocessing step for functions and predicates, that simplifies unification and lets us easily reuse existing indexing techniques. We have implemented the calculus in a prototype and first results are encouraging. Even though polymorphic encodings solve a harder problem, our evaluation shows that it they are competitive with the monomorphic encoding; itself a highly efficient encoding. With increasing number of axioms, the native polymorphic+typeclass runtime behavior performs best. We plan to use the typed calculus as a basis to bring further features of interactive theorem proving to the superposition calculus.

\section{References}

[1] F. Baader and T. Nipkow. Term Rewriting and All That. Cambridge University Press, New York, NY, USA, 1998.

[2] M. Baaz, U. Egly, and A. Leitsch. Normal form transformations. In Robinson and Voronkov [12, pages $273-333$. 
[3] L. Bachmair and H. Ganzinger. Rewrite-based equational theorem proving with selection and simplification. J. Log. Comput., 4(3):217-247, 1994.

[4] J. C. Blanchette, S. Böhme, and L. C. Paulson. Extending sledgehammer with smt solvers. J. Autom. Reasoning, 51(1):109-128, 2013.

[5] J. C. Blanchette, S. Böhme, A. Popescu, and N. Smallbone. Encoding monomorphic and polymorphic types. In N. Piterman and S. A. Smolka, editors, TACAS, volume 7795 of LNCS, pages 493-507. Springer, 2013.

[6] J. C. Blanchette and A. Paskevich. Tff1: The tptp typed first-order form with rank-1 polymorphism. In M. P. Bonacina, editor, CADE, volume 7898 of $L N C S$, pages 414-420. Springer, 2013.

[7] J. C. Blanchette, A. Popescu, D. Wand, and C. Weidenbach. More spass with isabelle. In L. Beringer and A. Felty, editors, Interactive Theorem Proving, volume 7406 of LNCS, pages 345-360. Springer Berlin Heidelberg, 2012.

[8] F. Bobot, S. Conchon, E. Contejean, and S. Lescuyer. Implementing Polymorphism in SMT solvers. In C. Barrett and L. de Moura, editors, SMT 2008: 6th International Workshop on Satisfiability Modulo, volume 367 of ACM International Conference Proceedings Series, pages 1-5, 2008.

[9] F. Bobot and A. Paskevich. Expressing polymorphic types in a many-sorted language. In C. Tinelli and V. Sofronie-Stokkermans, editors, FroCoS, volume 6989 of LNCS, pages 87-102. Springer, 2011.

[10] J.-F. Couchot and S. Lescuyer. Handling polymorphism in automated deduction. In F. Pfenning, editor, CADE, volume 4603 of $L N C S$, pages 263-278. Springer, 2007.

[11] P. Graf. Substitution tree indexing. In J. Hsiang, editor, Rewriting Techniques and Applications, volume 914 of LNCS, pages 117-131. Springer Berlin Heidelberg, 1995.

[12] J. A. Robinson and A. Voronkov, editors. Handbook of Automated Reasoning (in 2 volumes). Elsevier and MIT Press, 2001.

[13] S. Schulz. Simple and efficient clause subsumption with feature vector indexing. In M. Bonacina and M. Stickel, editors, Automated Reasoning and Mathematics, volume 7788 of LNCS, pages 45-67. Springer Berlin Heidelberg, 2013.

[14] C. Weidenbach. Combining superposition, sorts and splitting. In Robinson and Voronkov [12, pages $1965-2013$.

[15] M. Wenzel. Type classes and overloading in higher-order logic. In E. L. Gunter and A. P. Felty, editors, TPHOLs, volume 1275 of LNCS, pages 307-322. Springer, 1997. 